\title{
Single nucleotide polymorphism in wheat chromosome region harboring $F h b 1$ for Fusarium head blight resistance
}

\author{
Amy N. Bernardo $\cdot$ Hongxiang Ma $\cdot$ \\ Dadong Zhang • Guihua Bai
}

Received: 10 December 2010/ Accepted: 9 March 2011

(C) Springer Science+Business Media B.V. (outside the USA) 2011

\begin{abstract}
Fusarium head blight (FHB) is a destructive disease that reduces wheat grain yield and quality. To date, the quantitative trait locus on 3BS ( $F h b l)$ from Sumai 3 has shown the largest effect on FHB resistance. Single nucleotide polymorphism (SNP) is the most common form of genetic variation and is suitable for high-throughput marker-assisted selection (MAS). We analyzed SNPs derived from 23 wheat expressed sequence tags (ESTs) that previously mapped near Fhbl on chromosome 3BS. Using 71 Ning 7840/Clark BC7F7 recombinant inbred lines and the single-base extension method, we mapped seven SNP markers between Xgwm533 and Xgwm493, flanking markers for $F h b 1$. Five of the SNPs explained
\end{abstract}

Electronic supplementary material The online version of this article (doi:10.1007/s11032-011-9565-y) contains supplementary material, which is available to authorized users.

A. N. Bernardo

Department of Plant Pathology, Kansas State University, Manhattan, KS 66506, USA

H. Ma

Plant Genetics and Biotechnology, JAAS, Nanjing, China

H. Ma · D. Zhang · G. Bai

Department of Agronomy, Kansas State University,

Manhattan, KS 66506, USA

G. Bai $(\bowtie)$

Hard Winter Wheat Genetics Research Unit, USDA-ARS, Manhattan, KS 66506, USA

e-mail: gbai@ksu.edu
$45-54 \%$ of the phenotypic variation for FHB resistance. Haplotype analysis of 63 wheat accessions from eight countries based on SNPs in EST sequences, simple sequence repeats, and sequence tagged sites in the Fhbl region identified four major groups: (1) US-Clark, (2) Asian, (3) US-Ernie, and (4) Chinese Spring. The Asian group consisted of Chinese and Japanese accessions that carry Fhbl and could be differentiated from other groups by marker Xsnp3BS11. All Sumai 3-related accessions formed a subgroup within the Asian group and could be sorted out by Xsnp3BS-8. The SNP markers identified in this study should be useful for MAS of $F h b 1$ and fine mapping to facilitate cloning of the $F h b 1$ resistance gene.

Keywords Single nucleotide polymorphism . Fhbl · Fusarium graminearum · Marker-assisted selection - Genetic diversity

\section{Introduction}

Wheat Fusarium head blight (FHB), caused primarily by Fusarium graminearum, is a destructive wheat disease that reduces not only grain yield but also grain quality (Schroeder and Christensen 1963). Additional FHB-related losses result from contamination of grains with mycotoxins, such as deoxynivalenol, that are harmful to animal and human health (Desjardins and Hohn 1997; Snijders 1990). Several epidemics in North America resulted in an estimated yearly loss of 
at least 100 million bushels for the years 1991, 1993, and 1994 (McMullen et al. 1997). Use of FHBresistant cultivars is one of the most practical and effective solutions for minimizing FHB damage.

The Chinese variety Sumai 3 and its derivatives, which include Ning 7840, have a high level of FHB Type II resistance (resistance to symptom spread within a spike) and have been widely used as resistant parents in breeding programs worldwide (Wilcoxson et al. 1992). Sumai 3 inherited some FHB resistance quantitative trait loci (QTL) from the Chinese landrace Taiwan Wheat (Bai et al. 2003) and sequentially passed them to Ning 7840, a breeding line that also has resistance to three rust diseases and powdery mildew and other improved agronomic traits (Bai et al. 1989).

Fusarium head blight phenotyping is time-consuming, laborious, and often influenced by the environment (Andersen 1948). Moreover, reliable estimates of FHB resistance require evaluation of FHB symptoms in replicated experiments in several years and locations (Mesterhazy 1977). The difficulties associated with FHB phenotyping make it a beneficial trait for markerassisted selection (MAS). QTL for FHB resistance have been mapped in all wheat chromosomes except 7D (Buerstmayr et al. 2009). The most consistent QTL with the largest effect on Type II FHB resistance identified to date, $F h b l$, has been mapped on chromosome 3BS of Sumai3 and its derivatives; this QTL explained up to $53 \%$ of the phenotypic variation for FHB resistance and also facilitated low DON accumulation in infected kernels of Ning 7840 (Bai et al. 1999; Zhou et al. 2002a). Restriction fragment length polymorphism, simple sequence repeat (SSR), amplified fragment length polymorphism, and sequence tagged site (STS) markers closely linked to this QTL have been reported (Bai et al. 1999; Cuthbert et al. 2006; Liu and Anderson 2003; Waldron et al. 1999; Zhou et al. 2002b). Among all of these markers, Xgwm533 and Xgwm493, flanking markers for Fhbl, have been routinely used for MAS of Fhbl. However, these markers still define a relatively large chromosome interval for the QTL; more markers closely linked to the QTL are urgently needed to improve selection efficiency.

Single strand conformation polymorphism (SSCP) and single feature polymorphism (SFP) markers around the $F h b 1$ region in 3BS have recently been identified (Bernardo et al. 2009; Yu et al. 2008a). These expressed sequence tag (EST)-based markers detect polymorphisms by identifying DNA fragments that potentially contain DNA sequence variation, but complicated procedures for detection of these markers make them impractical for use in MAS. Single nucleotide polymorphisms are the most common form of genetic variation ( $\mathrm{Zhu}$ et al. 2003). The biallelic nature of SNPs coupled with their abundance and the availability of various flexible SNP genotyping platforms make SNPs an ideal future marker system for MAS (Koebner and Summers 2003; Rafalski 2002). Large EST databases have been developed for wheat and successfully mined for SNPs using contig alignments and/or resequencing (Mochida et al. 2004; Somers et al. 2003). Recently, an Illumina GoldenGate platform was used to analyze 96 SNPs in tetraploid and hexaploid wheat (Akhunov et al. 2009). However, the whole genome sequence of wheat may not be available in the near future, and the number of SNPs available for marker analysis in wheat is still limited. Furthermore, many SNPs in the Wheat SNP database (http://wheat.pw.usda.gov/SNP/ new/index.shtml) were developed from wild wheat relatives.

In this study, we developed SNP markers linked to Fhbl on the basis of EST sequences mainly from previously published SSCP and SFP markers in the Fhb1 region (Bernardo et al. 2009; Yu et al. 2008a) to facilitate high-throughput MAS and high-resolution mapping. The SNPs were mapped in a BC7F7 population from the cross of Ning 7840/Clark to identify the closest SNP markers for $F h b 1$. The haplotype diversity of mapped ESTs was also examined.

\section{Materials and methods}

Mapping population

A BC7F7 recombinant inbred line population was developed for fine mapping of $F h b 1$ by backcrossing Clark to Ning 7840 seven times (Electronic Supplementary Material Fig. 1). Ning 7840 (Aurora/Anhui// Sumai3) is a hard red facultative Chinese wheat line with a high level of resistance to FHB. Clark [Beau// (65256A1-8-1/67137B5-16/4/Sullivan/3/Beau//5517B 8-5-3-3/Logan)], released from Purdue University, IN, USA, is a soft red winter wheat cultivar highly susceptible to FHB. In the backcross scheme, each generation was subjected to MAS using two flanking 
SSR markers (Xgwm389/Xgwm533 and Xgwm493) and grown in the greenhouse at $25^{\circ} \mathrm{C}$ for $12 \mathrm{~h}$ of light and at $18^{\circ} \mathrm{C}$ for $12 \mathrm{~h}$ of darkness.

Marker analysis and recombinant selection

After seven backcrosses, 1,750 BC7F2 plants were screened with Xgwm389 and Xgwm493, and plants that showed recombination between the two markers were selected for advancing to further generation. Starting from the BC7F5, selected families were evaluated for FHB resistance and genotyped with Xgwm533 and Xgwm493, markers that flanked a shorter Fhbl-containing fragment. The SSR markers were visualized using fluorescence-labeled M13 primer in conjunction with M13-tailed primer for PCR, and amplicons were separated in a capillary DNA analyzer (Sun et al. 2009). Selected lines with recombination between the markers Xgwm533 and Xgwm493 were evaluated for FHB resistance using the single floret inoculation method. $F$. graminearum conidia (strain GZ 3639, a Kansas field isolate) were produced as described by Bai et al. (2000). At anthesis, $10 \mu \mathrm{l}$ ( $\sim 1,000$ spores $)$ of $F$. graminearum conidiospore suspension was injected into the floral cavity between the lemma and palea of a floret in the middle of a spike. Inoculated plants were temporarily housed in an enclosed plastic chamber with $100 \%$ humidity for 3 days to initiate FHB infection. Disease spread within an inoculated spike was evaluated as the percentage of infected florets in a spike (number of infected florets divided by the total number of florets in a spike) 15 (summer) to 18 (winter) days after inoculation, depending on the FHB evaluation season. All experiments were designed in randomized complete blocks with at least three replicates (pots) of five plants per line in each experiment. About five spikes at similar developmental stage were inoculated per pot. FHB symptoms were evaluated for three generations (BC7F4-5, BC7F5-6 and BC7F5-7) during 2007 and 2008 in greenhouses at Kansas State University, Manhattan, KS. USA. Ning 7840 and Clark were included as resistant and susceptible checks in each experiment.

\section{SNP marker development}

Genomic DNA from Ning 7840 and Clark was extracted from leaf tissues following the CTAB protocol (Saghai-Maroof et al. 1984). To generate PCR fragments for sequencing, primer pairs were designed according to (1) the Affymetrix wheat consensus EST sequence of 19 SFP markers that were mapped within or near the marker interval of Xgwm533-Xgwm493 (Bernardo et al. 2009) and (2) the EST sequence of three SSCP markers (Yu et al. 2008b). Primers were designed using the computer program PerlPrimer v1.1.9 (Marshall 2004). Umn10 primers for an STS marker near Fhbl were also used to generate PCR products (Liu et al. 2008). The PCR mix contained $1 \times \mathrm{NH}_{4}$ buffer (Bioline, Randolph, MA, USA), $2 \mathrm{mM} \mathrm{MgCl}_{2}, 0.2 \mathrm{mM}$ dNTPs, $0.5 \mu \mathrm{M}$ of each primer, $100 \mathrm{ng}$ DNA, and 1 unit Taq polymerase (Promega, Madison, WI, USA) in a reaction volume of $12 \mu \mathrm{l}$. PCR was carried out in a PTC-100 thermal cycler (MJ Research, Inc.) using a touchdown program starting at $94^{\circ} \mathrm{C}$ for $5 \mathrm{~min}$; followed by 10 cycles of $20 \mathrm{~s}$ at $94^{\circ} \mathrm{C}, 20 \mathrm{~s}$ at $63^{\circ} \mathrm{C}$ minus $0.5^{\circ} \mathrm{C}$ per cycle, and $2 \mathrm{~min}$ at $72^{\circ} \mathrm{C} ; 35$ cycles of $20 \mathrm{~s}$ at $94^{\circ} \mathrm{C}, 20 \mathrm{~s}$ at $58^{\circ} \mathrm{C}$, and $2 \mathrm{~min}$ at $72^{\circ} \mathrm{C}$; and a final extension of $10 \mathrm{~min}$ at $72^{\circ} \mathrm{C}$. Polymerase chain reaction products were visualized in a $2 \%$ agarose gel, and singletons were selected for sequencing. Selected PCR products $(5 \mu \mathrm{l})$ were treated with $1.7 \mathrm{U}$ shrimp alkaline phosphatase (SAP) and 0.7 U Exonuclease I (USB Corporation, Cleveland, OH, USA), incubated at $37^{\circ} \mathrm{C}$ for $1 \mathrm{~h}$, and then deactivated at $75^{\circ} \mathrm{C}$ for $15 \mathrm{~min}$. DNA sequencing was done using an ABI 3730 DNA Analyzer and BigDye Terminator V1.1 (Applied Biosystems, Foster City, CA, USA) at the Kansas State University Sequencing and Genotyping Facility. DNA sequence data of Ning 7840 and Clark were viewed using DNA Sequencing Analysis Software v5.2 (Applied Biosystems) and manually checked for SNPs.

Following the SNaPshot Kit primer design guidelines (Applied Biosystems), SNP primers were designed as 18-24 bases long with the $3^{\prime}$ end of the primer ending right before the SNP. All primers were checked for possible extendable primer-dimer formation using PerlPrimer v1.1.9 (Marshall 2004). Single nucleotide polymorphisms were analyzed with the SNaPshot kit according to the manufacturer's manual. The template used for SNP primer extension was generated by PCR, and PCR cleanup was done using the SAP-ExoI treatment described above. A second PCR was performed in a $10 \mu \mathrm{l}$ reaction with $1 \mu \mathrm{l}$ SNaPshot multiplex ready reaction mix, $0.2 \mu \mathrm{M}$ 
primer, and $0.5 \mu \mathrm{l}$ of the first PCR product as template. Samples were subjected to 25 cycles of $96^{\circ} \mathrm{C}$ for $10 \mathrm{~s}, 56$ or $58^{\circ} \mathrm{C}$ (depending on the annealing temperature of the primer) for $5 \mathrm{~s}$ and $60^{\circ} \mathrm{C}$ for $30 \mathrm{~s}$ in a PTC-100 thermal cycler (Bio-Rad, Hercules, CA, USA). After PCR products were incubated with 1 U SAP (USB Corporation, Cleveland, OH, USA) to remove unincorporated dNTPs, PCR products $(0.5 \mu \mathrm{l})$ were mixed with $\mathrm{Hi}$-Di formamide and GeneScan 120 Liz size standard (Applied Biosystems). Electrophoresis was done on a 3730 DNA Analyzer (Applied Biosystems), and data were scored using GeneMarker software (Soft Genetics, State College, PA, USA). All SNP-marker-allele calls from Ning 7840 and Clark were compared with the expected allele calls from DNA sequencing to identify polymorphisms between Ning 7840 and Clark (Supplementary Table 1). For functional annotation, sequences were compared with the nonredundant protein database of NCBI of June 2009 using BLASTX (Altschul et al. 1990). Sequence similarity was considered significant if $E \leq 0.001$.

Genetic mapping and QTL analysis

A set of 71 selected BC7F7 lines was used for fine mapping. One seed per line was planted in a $5 \times 5$ inch $(12.7 \times 12.7 \mathrm{~cm})$ Dura-pot (Hummert Int., St. Louis, MO, USA) containing Metro Mix 360 soil mix (Hummert Int.) and grown in a growth chamber at $4^{\circ} \mathrm{C}$ under $12 \mathrm{~h}$ of light and $12 \mathrm{~h}$ of darkness. Leaves were collected in 1.1-ml 8-strip microtubes (ISC BioExpress, Kaysville, UT, USA) when plants reached the two-leaf stage. Collected tissues were lyophilized for 2 days in a ThermoSavant Modulyo D-115 freeze dryer (Thermo Scientific, Waltham, MA, USA). To grind leaf tissues into a fine powder, a 4.5-mm Copperhead 6000 premium BBS (Crosman Corp., E. Bloomfield, NY, USA) was added into each tube, and the tubes were shaken for $3 \mathrm{~min}$ at a frequency of 20 times per second in a Mixer Mill 300 (Retsch, Inc., Newton, PA, USA). Ground samples were stored at $-80^{\circ} \mathrm{C}$ until DNA extraction.

Seventeen markers (nine SNPs, four STS, and four SSRs) were analyzed in the population. The STS markers on 3BS that were previously mapped near the Fhbl region (Liu and Anderson 2003; Cuthbert et al. 2006) were also analyzed using the SSR protocol (Sun et al. 2009). Marker data were scored as $A$ for Ning 7840 allele, $B$ for Clark allele, and $H$ for heterozygous alleles. To ensure accurate scoring, all markers were scored at least twice. Loci with ambiguous bands were not scored. All markers were mapped using JoinMap 3.0 (Kyazma, Wageningen, Netherlands) with the Kosambi mapping function (Kosambi 1944) and an LOD of three to declare a significant QTL. Composite interval mapping was used to map the QTL with QGene 4.0 (Roby and Nelson 2008).

Haplotype analysis

A set of 62 different wheat varieties and breeding lines from eight countries were evaluated previously for Type II FHB resistance and pedigree information (Bai et al. 2003), and thus used for validation of SNPs in this study. Genomic DNA from those 62 and Chinese Spring was amplified using PCR primers for seven SNP markers (Xsnp3BS-3 to Xsnp3BS-8, and Xsnp11). Amplified PCR products were sequenced. DNA sequences in FASTA format were imported into Biology Workbench 3.2 (San Diego Supercomputer Center, University of California, San Diego, CA, USA) and aligned using ClustalW (Thompson et al. 1994). The SNP alleles were converted to binary data (i.e., a score of 1 if Ning 7840-like and 0 if otherwise). Neighboring SNP alleles with similar scores within an amplicon were grouped into haplotype blocks to reduce redundancy. The 63 different lines were also genotyped using SSR markers Xgwm533, Xgwm493, and Xbarc147. The presence or absence of a band of the same size was assumed as alleles of a locus, and bands were given a score of 1 when present and 0 for absence. Binary data scores from all markers were combined, and a Dice similarity coefficient matrix was constructed for each pair of wheat lines using SIMQUAL module (NTSYSpc V2.02i, Exeter Software, Setauket, NY, USA). The SAHN module and unweighted pair group method with arithmetic mean was used for cluster analysis (Rohlf 1998).

\section{Results}

SNPs between parents

To identify SNPs, DNA from Ning 7840 and Clark was amplified with primers designed on the basis of 23 EST 
sequences that harbor either SFP or SSCP. Singletons and high-quality DNA sequences were generated from both parents for $13 \mathrm{EST}$. Of the $13 \mathrm{EST}$ sequences, 11 contained 145 SNPs, 31 indels, and 17 multiple nucleotide polymorphisms (adjacent SNPs) between Ning 7840 and Clark, and two were monomorphic. For the other 10 ESTs, quality sequencing failed in at least one parent, even after three or more different primer pairs were tested. Among them, one sequence from Ning 7840 (Ta.24841.2.S1) had no significant homology with the corresponding sequence from Clark and the Affymetrix EST sequence and therefore was not analyzed further; six ESTs generated good sequences for only one parent (no amplification in the other); and the three others showed either multiple or no bands in both parents.

\section{SNP markers in Fhbl region}

Two to three SNP primers per gene were designed and tested for the 11 SNP-containing genes, and one SNP per gene was selected for mapping. All amplified SNP alleles matched with the sequence data of Ning 7840 and Clark (Table 1). Nine SNP together with four SSR and four STS markers were mapped in the linkage group of $F h b 1$ on 3BS using the BC7F7 population. Among the nine SNPs, seven were mapped within the $F h b 1$ region flanked by Xgwm533

Table 1 Coefficients of determination $\left(R^{2} \times 100\right)$ and LOD of molecular markers in the $F h b 1$ region calculated from the population derived from Ning 7840/Clark

\begin{tabular}{|c|c|c|}
\hline Marker & $R^{2}(\%)$ & LOD \\
\hline Xgwm533 & 11.50 & 1.84 \\
\hline Xsnp3BS-3 & 11.60 & 1.84 \\
\hline Xbarc147 & 48.70 & 10.00 \\
\hline Xsts3B-163 & 53.50 & 11.47 \\
\hline Xsnp3BS-5 & 53.50 & 11.47 \\
\hline Xsnp3BS-4 & 53.90 & 11.61 \\
\hline Xsnp3BS-11 & 54.00 & 11.62 \\
\hline Xsts3B-138 & 54.00 & 11.62 \\
\hline Xsts256 & 46.00 & 9.21 \\
\hline Xsnp3BS-7 & 45.20 & 9.02 \\
\hline Xsnp3BS-8 & 48.60 & 9.96 \\
\hline Xsts3B-142 & 23.00 & 3.91 \\
\hline Xsnp3BS-6 & 11.10 & 1.76 \\
\hline Xgwm493 & 10.70 & 1.70 \\
\hline
\end{tabular}

and Xgwm493 (Fig. 1), and five were mapped in a marker-dense region with four other markers covering a genetic distance of $1.1 \mathrm{cM}$. This region gave the highest $R^{2}(45-54 \%)$ and LOD values (9.02-11.62) and is the most likely location of Fhbl (Table 1; Fig. 2). Markers Xsts $3 B-138$ and Xsnp3BS- 11 had the largest average $R^{2}$ value. SNPs $X s n p 3 B S-1$ and Xsnp3BS-2 were mapped between Xgwm389 and Xgwm533 toward the distal end of chromosome 3BS, and Xsnp3BS-9 was mapped outside of Xgwm493 (toward the centromere). Xsnp3BS-10 was monomorphic and therefore was not mapped.

Eight different types of recombinants (Table 2) were identified among the BC7F7 lines after 17 markers on 3BS were genotyped (Fig. 1). Recombinant inbred lines with recombinant types 4,5 , and 6 showed the resistant phenotype in the greenhouse conditions. These resistant lines had seven common markers: Xbarc147, Xsts163, Xsnp5, Xsnp4, Xsnp11, $X s t s 138$, and Xsts256. Two type-6 recombinants had Ning 7840 alleles for markers covering Xgwm533 to Xsts256 and Clark alleles from Xsnp7 to Xgwm493; one exhibited the resistant phenotype, and the other was only moderately resistant. On the other hand, a type-2 recombinant with 3BS marker alleles completely opposite to type- 6 showed moderate susceptibility to FHB.

Fig. 1 Fine genetic map of the wheat Fhb1 3BS chromosome region in the Ning 7840/Clark BC7F7 population. Numbers to the left of the vertical bar are interval distances in centimorgans, and mapped markers are on the right

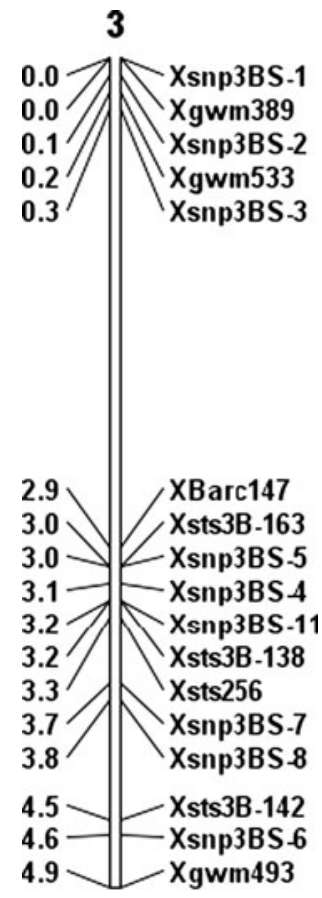


Fig. 2 Interval analysis of chromosome 3BS for FHB resistance in the Ning 7840/Clark mapping population. LOD values $(y$ axis) were determined from three separate FHB phenotyping experiments. Marker names in the $x$-axis are listed according to map order and distance

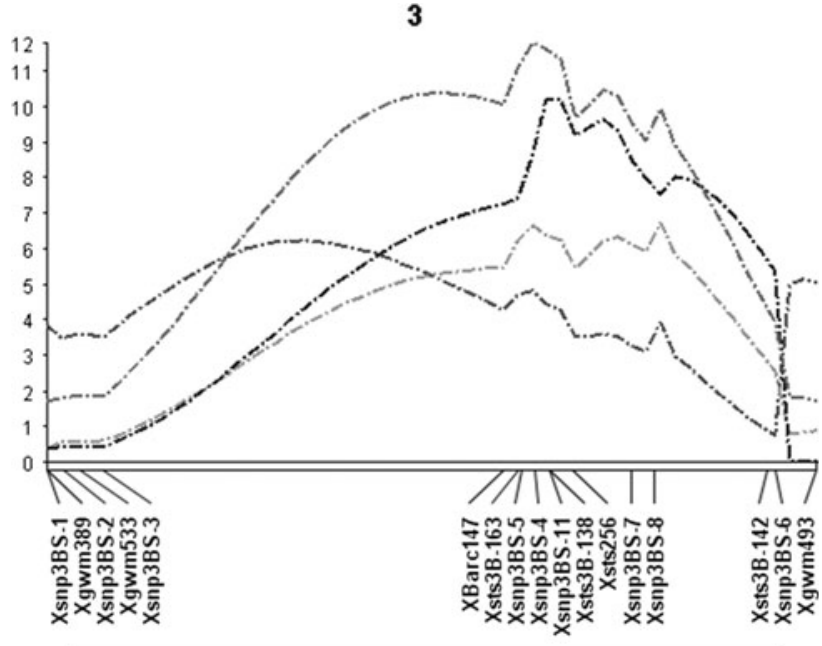

Spring-2007 --- LOD, Composite IM (LS) Fall-2007

Table 2 Genotypes and phenotype of selected recombinants

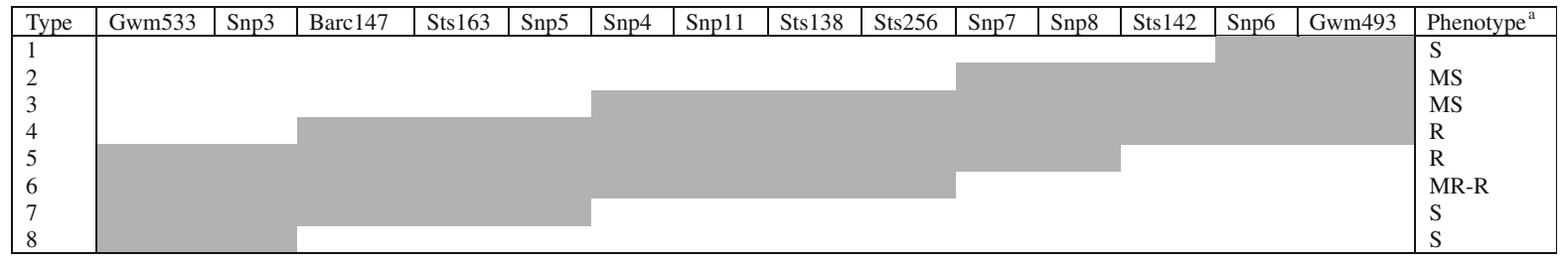

Gray-shaded boxes denote Ning 7840-like allele for that particular marker; unshaded boxes denote Clark-like allele

a $S$ susceptible, $M S$ moderately susceptible, $R$ resistant, $M R$ moderately resistant

Functional annotation of EST

A sequence homology search of the seven EST sequences in the nonredundant protein database of GenBank identified only one EST (Snp3BS-3) with a putative defense-related function; this EST had significant homology with a rice NBS-LRR disease resistance protein. Two mapped ESTs in the delimited region significantly matched $\left(E \leq 10^{-3}\right)$ with hypothetical proteins of unknown function, and four had no similarity with any protein in the NCBI database.

Haplotype of Fhbl marker alleles in other wheat germplasm

To investigate the distribution frequency of $F h b 1$ marker alleles that mapped in the Ning 7840/Clark population, the SNP-containing EST sequences were analyzed across 63 diverse germplasm accessions together with SSR and STS markers in the Fhbl region. These accessions showed a wide range of head blight severity (Bai et al. 2003). Cluster analysis identified four major clusters (Fig. 3).

The first cluster had eight accessions with five from the USA (including the FHB-susceptible Clark) and one each from Brazil (Encruzilhada), Austria (Expert), and China (NTDPH). The US breeding line P93D1-10-2 and Encruzilhada have the same alleles as Clark for all markers tested. The other accessions in this cluster have Clark-like haplotype/alleles for five (NTDHP) to ten markers (Expert); this cluster is therefore called the Clark cluster. Wheat response to FHB infection (PSS) for accessions in this cluster ranged from $17 \%$ (resistant) for P93D1-10-2 to $94 \%$ (susceptible) for Clark (Bai et al. 2003).

The second cluster (Asian cluster) consisted of 19 accessions from China and Japan and one (IL963424851) from the USA (Fig. 3; Bai et al. 2003). Eight 


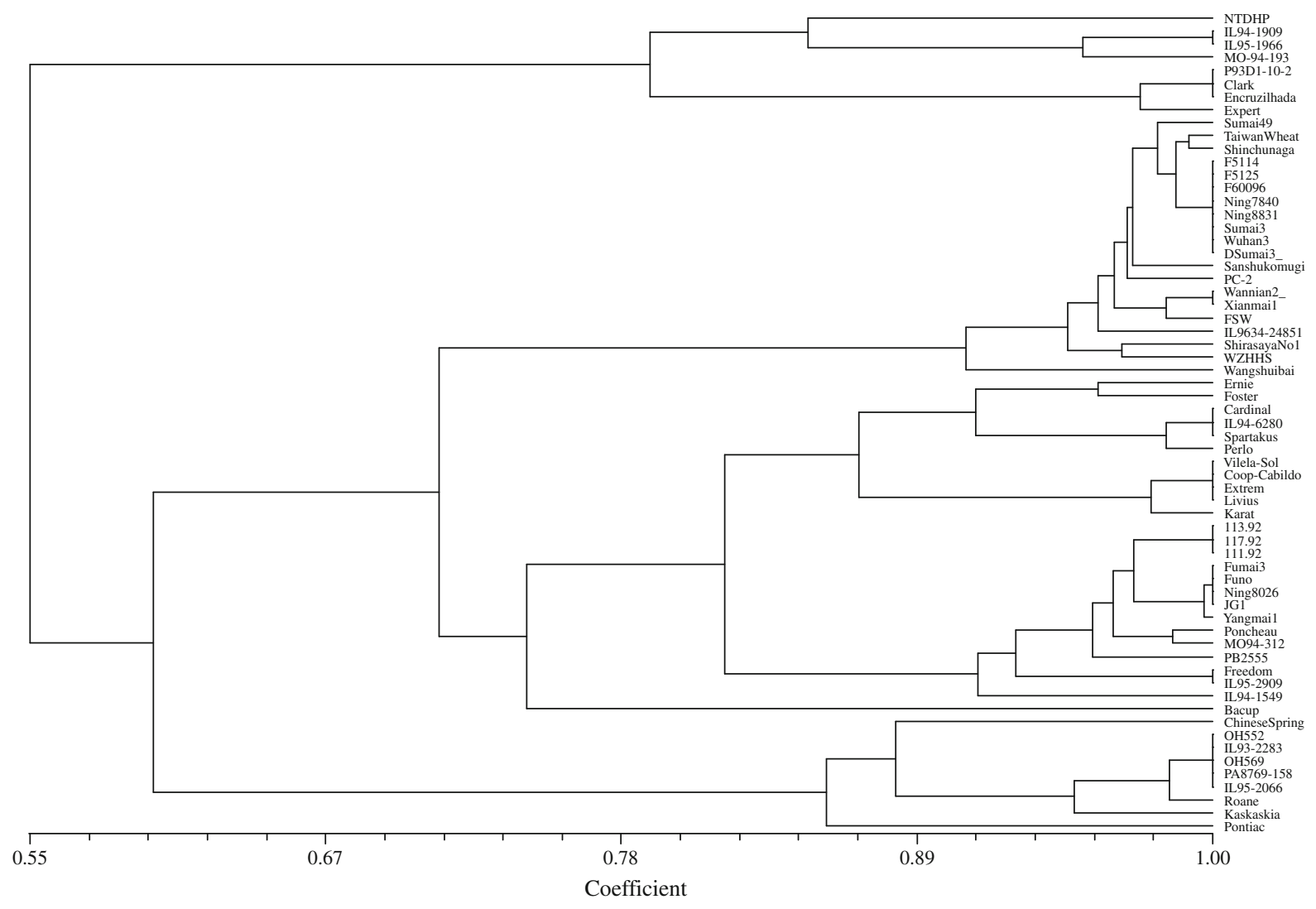

Fig. 3 Unweighted pair group method with arithmetic mean dendogram resulting from Dice similarity coefficient matrix showing the genetic relationship of 63 accessions based on EST sequence (SNP), SSR, and STS markers associated with Fhb1

accessions with either Sumai 3 or Ning 7840 in their pedigrees showed the same genotype as Taiwan Wheat (one parent of Sumai 3) and formed the Ning 7840 subcluster. Accessions F5114, F5125, F60096, Ning 8331, Sumai 3, Wuhan 3, and DSumai 3 carried the Ning 7840 alleles for all markers tested. Taiwan Wheat showed the same marker profile as the Ning 7840 subgroup except for a haplotype difference in the Snp3BS-4-containing DNA fragments (Supplementary Table 2). Shinchunaga from Japan had all of the same marker alleles as Taiwan Wheat and therefore was also included in the Ning 7840 subgroup. Sumai 49 had alleles from two markers (Xgwm389 and Xgwm533) that differed from Ning 7840 and is therefore clustered closer to Taiwan Wheat than to Ning 7840 or Sumai 3, although Sumai 3 is one of its parents. These two SSR markers are close to the distal border of Fhbl (Fig. 1) and may not be tightly linked markers for FHB Type II resistance; thus, accessions in this subcluster most likely carry Fhbl. Eight out of the eleven accessions in this subcluster showed a high level of Type II resistance with PSS $\leq 13 \%$, and the other three accessions (F60096, Ning8831 and Wuhan3) showed moderate FHB resistance with PSS from 0.21 to 0.36 (Bai et al. 2003).

Another Asian subcluster consisted of accession IL9634-24851, five Chinese accessions and two Japanese landraces (Fig. 3). These accessions mainly differed from the Ning 7840 group in the Snp3BS-8 haplotype and also in Snp3BS-4 and Xgwm493 alleles in most cases. However, they have most of the Ning 7840 marker alleles in the Fhbl region and thus likely carry $F h b 1$, but might be a different allele. IL9634-24851 has Ning 7840 in its pedigree, and thus is classified in this group. Wangshuibai branched at $90 \%$ similarity and can be loosely classified into this subgroup. Most accessions in this subcluster showed resistance or moderate resistance to FHB infection with PSS from 13\% (WZHHS) to 52\% (Xianmai) (Bai et al. 2003). 
Chinese Spring, an accession moderately susceptible to FHB with PSS of $60 \%$ (Jayatilake et al. 2011), and eight US accessions belong to the third major group. This group had the Ning 7840 haplotypes/alleles for only three to four markers/ESTs (Snp3BS-3, Snp3BS-6, Snp3BS-7, and Xgwm493) but carried haplotypes/alleles different from those of Ning 7840 and Clark for all other markers (Fig. 2 and Supplementary Table 2). Xsnp3BS-3 mapped close to Xgwm533 and was located on the valley of the 3BS QTL map; therefore, it may not be a tightly linked marker for Fhb1. Similarly, Xsnp3BS-7, Xsnp3BS-6, and $X g w m 493$ are close to the proximal border of $F h b 1$ (Fig. 1) and are relatively far from $F h b 1$; thus, they may not be critical markers for Fhbl. Therefore, it is unlikely that this group carries the Fhbl resistance allele. The accessions in this cluster had various levels of resistance to FHB, with PSS from 14 to $71 \%$ Bai et al. (2003).

The fourth cluster, which was also the largest cluster, consisted of 26 lines originating from Austria, France, USA, Argentina, and China. Accessions in this cluster did not have any fixed pattern of markerallele combinations and showed mixed alleles of Ning 7840, Clark, Chinese Spring, and others. These accessions had Ning 7840 marker alleles/haplotype for Xbarc147 and/or Snp3BS-5 (Supplementary Table 2). The Chinese accessions Fumai3, Ning 8026, and JG1 have Funo, a moderately susceptible FHB cultivar from Italy, in their pedigrees and Yangmai 1 was a selection of Funo. Yangmai 1 had one missing datum (Snp3BS-8) and did not cluster with the other Funo-related accessions. The moderately resistant accessions from the USA (Freedom and Ernie) and Austria (Karat, Extrem, Livius, Perlo, and Spartakus) are also included in this cluster. These accessions do not seem to have the Fhbl resistance allele, and most of the accessions in this cluster were moderately susceptible or susceptible to FHB. Only five accessions (Spartakus, Perlo, Poncheau, IL952909 and Bacup) have PSS of $14 \%$ or lower (Bai et al. 2003).

The number of SNPs was not correlated with the number of haplotypes identified in a specific EST (Table 3). For example, the EST sequence containing Snp3BS-3 had 55 SNPs that grouped into two haplotypes, whereas five different haplotypes were identified from 15 SNPs in the Snp3BS-6 fragment. By examining the diverse accessions and their corresponding SNP marker haplotypes, two SNP markers for Fhbl were identified: Snp3BS-11 differentiated the Chinese and Japanese accessions that carry Fhbl from other groups, and Snp3BS- 8 sorted out all Sumai 3-related accessions from all other accessions in the Asian group except that Shinchunaga and JG1 did not show any relationship with Sumai 3 according to available pedigree information.

\section{Discussion}

Single nucleotide polymorphisms are abundant and responsible for a majority of the phenotypic variation in plants (Kumar et al. 2007). This abundance provides a rich source of DNA markers for tagging genes of interest. Individual SNPs may also directly contribute to phenotypic variation if they are in an intra-genic or promoter region (Beales et al. 2005; Konishi et al. 2006) and can be used as "perfect" markers for selecting genes and traits of interests. SNPs have been successfully used to map genes or QTL in several species. For example, SNPs were reported to be associated with gene(s) for fragrance (Jin et al. 2003) and blast resistance (Hayashi et al.

Table 3 Number of SNPs, indels, and haplotypes identified in SNP-containing fragments in the 3BS FHB QTL region

\begin{tabular}{|c|c|c|c|c|c|c|c|c|c|}
\hline Number of & Snp3BS-1 & Snp3BS-2 & Snp3BS-3 & Snp3BS-4 & Snp3BS-5 & Snp3BS-6 & Snp3BS-7 & Snp3BS-8 & Snp11 \\
\hline SNPs & 1 & 16 & 55 & 24 & 10 & 15 & 5 & 5 & 8 \\
\hline MNPs (adjacent SNPs) & 0 & 1 & 13 & 1 & 0 & 2 & 2 & 0 & 2 \\
\hline Indels & 3 & 7 & 6 & 4 & 0 & 6 & 1 & 1 & 4 \\
\hline Haplotypes & $\mathrm{ND}^{\mathrm{a}}$ & $\mathrm{ND}^{\mathrm{a}}$ & 2 & 4 & 4 & 5 & 2 & 3 & 5 \\
\hline SNPs in haplotype-tag & $\mathrm{ND}^{\mathrm{a}}$ & $\mathrm{ND}^{\mathrm{a}}$ & 1 & 3 & 3 & 4 & 1 & 2 & 4 \\
\hline Accessions scored & 2 & 2 & 44 & 58 & 60 & 58 & 54 & 42 & 61 \\
\hline
\end{tabular}

${ }^{\text {a }} N D$ not determined 
2004) in rice, pungency in pepper (Garcés-Claver et al. 2007), and nematode resistance in potato (Sattarzadeh et al. 2006) and soybean (Ha et al. 2007).

In this study, ESTs that mapped around the Fhbl region in chromosome $3 \mathrm{BS}$ were sequenced to identify SNPs between Ning 7840 and Clark. Among these ESTs, three were previously characterized as SSCP markers (Yu et al. 2008a), 19 as SFPs (Bernardo et al. 2009), and one as an STS marker (Liu et al. 2008). However, SSCP does not provide polymorphic sequence information and marker detection usually uses nondenaturing polyacrylamide gel electrophoresis that is not suitable for automation. Furthermore, SSCP is affected by the tendency of single-stranded DNA to adopt several conformational forms even when electrophoretic conditions are the same (Schwieger and Tebbe 1998), so detecting variations is laborious and has low sensitivity. Although SFP analysis uses a high-throughput platform, it is associated with the expensive Affymetrix Wheat Genome Arrays analysis, which makes it impractical for genotyping a few markers in many samples, and some SFPs may be associated with an RNA expression level in which the polymorphism between parents cannot be traced to the DNA level. Single nucleotide polymorphism analysis can detect specific polymorphic alleles responsible for the sequence variation; it thus provides more accurate genotyping than SSCP and SFP analyses. Moreover, SNPs are amenable to automation and are therefore more suitable for high-throughput genotyping and large-scale MAS.

Several STS and SSR markers have already been mapped between Xgwm533 and Xgwm493; however, the map is far from saturated. To fine-map Fhbl, lines having recombination between Xgwm389 and Xgwm493 were selected starting from the BC7F2 population. The region of recombination was further shortened to that between Xgwm533 and Xgwm493 at the BC7F7 population. The Fhbl region was saturated with seven EST-derived SNP markers (Fig. 1). Two type-6 recombinants had Ning 7840 alleles for markers covering Xgwm533 to Xsts256 and Clark alleles from Xsnp3BS-7 to Xgwm493: one had the resistant phenotype, and the other was only moderately resistant. This implies that the recombination breakpoints between Xsts256 and its adjacent marker Xsnp7 may be different between these two lines and that the distal border of Fhbl lies between these two markers in our population. The existence of a moderately susceptible line having Clark alleles from Xgwm533 to Xsts256 and the Ning 7840 fragment for markers Xsnp7 to Xgwm493 (type 2) lends further support to this border designation. The proximal border of Fhbl cannot be determined from the recombinant types but the LOD value indicates that it is likely around Xbarcl47 (Table 1; Fig. 2). Five of the SNP markers gave LOD values ranging from 9.02 to 11.62 and could explain $45-54 \%$ of the phenotypic variation for FHB resistance. These markers should be better than Xgwm533 and Xgwm493, the SSR markers commonly used for MAS. Six STS markers were mapped between Xgwm533 and Xgwm493 in the Sumai 3/Stoa population, and the markers Xsts3B138 and $X s t s 3 B-52$ were co-localized in the same position and gave the highest $R^{2}$ and LOD values (Liu and Anderson 2003). However, Liu and Anderson cautioned that the marker order and QTL location may not be precise because of the small population size used. Using a larger population size, Cuthbert et al. (2006) mapped Fhbl and five of the STS markers previously developed by Liu and Anderson (2003) in a Sumai 3/Thatcher population. In our study, three of the six STS markers were mapped in the same order as reported by Cuthbert et al. (2006).

To determine the genetic diversity of the mapped ESTs and validate the SNPs, we sequenced the corresponding ESTs in 63 wheat accessions from different countries. DNA sequencing was chosen over SNP genotyping because sequencing would cover all SNPs in the ESTs (not only those polymorphic between Ning 7840 and Clark) and we wanted to obtain as much information as possible.

Genetic diversity analysis of EST (SNP), SSR, and STS markers associated with $F h b l$ generated four major groups: (1) Clark, (2) Asian, (3) Chinese Spring, and (4) Ernie. Three accessions in the Clark group (Clark, MO-94-193, and Encruzilhada) carry non-Ning 7840 genotypes for at least nine out of ten markers tested. Interestingly, Clark and MO-94-193 are very susceptible to FHB, whereas Encruzilhada has Type II resistance (Bai et al. 2003; Snijders 1990; Zhang et al. 2008). These results suggest that the accessions in this cluster most likely have susceptible alleles at the Fhbl locus.

In this study, the Asian group consisted mostly of Chinese and Japanese accessions and had at least 
eight (out of ten) genotypes that carried all Ning 7840-like marker alleles at the Fhbl region. The Japanese landrace Shinchunaga shared the same alleles with Taiwan Wheat, although one marker allele was different from that of Ning 7840. Thus, they all likely carry the same Fhbl as Ning 7840 . Although Wangshuibai, a Chinese FHB resistance landrace, had only five Ning 7840 marker alleles ( $90 \%$ similarity) and a different pedigree from Sumai 3, it may also carry the Fhbl locus, but a different allele, because a major QTL has been consistently mapped in the same location in several studies (Zhang et al. 2004; Zhou et al. 2004; Yu et al. 2008b).

Chinese Spring had similar marker alleles to soft red winter wheat accessions (e.g., OH552) at the Fhbl region and carried six to seven haplotypes/ alleles different from those of Ning 7840. Chinese Spring is a landrace and moderately susceptible to FHB. Whether it carries a resistance allele in the Fhbl locus remains a controversy (Grausgruber et al. 2004; Ma et al. 2006). Thus, the allele in Chinese Spring must be different from that of Ning 7840 if it carries a resistance allele at the Fhbl locus.

The Ernie group consisted of accessions from Europe, Argentina, USA, and China that carry a combination of Ning 7840, Clark/Encruzilhada, Chinese Spring, and other alleles. The Chinese accessions in this group (Fumai3, Ning 8026, JG1, and Yangmai1) are related to Funo, a moderately FHBsusceptible cultivar from Italy. These Funo-related accessions most likely do not possess a resistanceconferring allele at the 3BS QTL. Two US accessions in this group, Foster and Ernie, were reported to have a FHB resistance QTL, but not Fhbl (Liu et al. 2007; Sneller et al. 2004). Thus, the resistance of resistant accessions in this cluster is most probably controlled by a QTL other than Fhbl. Some resistant germplasm in this cluster may be a good source of resistance QTL for gene pyramiding with Fhbl to improve the level of resistance in cultivars.

Results of this study suggest that two SNP markers for Fhbl can be used for MAS: Xsnpl1 for Chinese and Japanese accessions with Fhbl and Xsnp3BS-8 for Sumai 3-related accessions carrying Fhbl. Snp3BS-8 could differentiate Sumai 3-related accessions from other accessions in the Asian group, except that Shinchunaga and JG1 appear to be unrelated to Ning 7840 on the basis of their pedigrees (Bai et al. 2003). Shinchunaga had similar alleles/ haplotypes with Taiwan Wheat for all markers tested and most likely has Fhbl. JG1 has only three Ning 7840-like marker alleles/haplotypes and does not belong to the Asian cluster. Nevertheless, these two SNP markers should be useful for MAS for Fhbl in breeding programs. Nine SNPs that were mapped in the Fhbl region will facilitate the removal of undesirable alleles near Fhbl (reduction in linkage drag) by MAS and also provide more alternate markers to use when some commonly used markers are nonpolymorphic between genotypes. The newly constructed map will also find application in fine mapping of Fhbl and facilitate map-based cloning and identification of the genes underlying FHB resistance.

The SNP conversion rate from SFP to SNP was relatively low (10/22) in this study, mainly because of failure in amplification of SNP-containing DNA fragments from both parents. A similar conversion rate was reported for the soybean Ragl aphid resistance gene (Kaczorowski et al. 2008). Wheat is a complex allopolyploid with three homeologous genomes and a huge genome size of $17,000 \mathrm{Mb}$, about $80 \%$ of which are repetitive sequences (Smith and Flavell 1975). The presence of three homeologous genomes complicates sequencing and SNP analysis. The biggest bottleneck encountered in this study was generating singletons for DNA sequencing. Even after several attempts, some of the primers designed from the wheat EST sequences could amplify DNA from only one or none of the parents. Primers from two SFPs did not amplify polymorphic sequences between Ning 7840 and Clark. Possible reasons for this could be that the primers designed in this study amplified a different homeolog or that both SFPs resulted from the differences in gene expression levels.

The SNPs found in this study were all biallelic. Because SNPs are mostly biallelic in nature, their polymorphism information content (PIC) is lower than that of SSR markers. To obtain the maximum information, haplotype-based genotyping (haplotypetag) is essential for a single locus with more than two haplotypes. However, assaying for more SNPs will also increase the cost of genotyping, so determination of the minimum number of SNPs that can differentiate all haplotypes is important for MAS. For example, the EST containing Snp3BS- 8 has five SNPs, but genotyping using two SNP markers 
(haplotype-tag) can differentiate all three haplotypes (Table 2). Although SNP markers are less informative than SSR markers, their abundance could compensate for their lower polymorphism information content.

Functional characterization of the EST-based SNP markers may determine candidate genes for FHB resistance. Snp3BS-3 had significant homology with a rice NBS-LRR disease resistance protein. However, this marker can explain only $7.71 \%$ of the phenotypic variation observed and likely does not play a role in FHB resistance. Other mapped ESTs in the delimited region showed homology with hypothetical proteins of unknown function or had no significant similarity with any protein in the NCBI database. Whether these genes play a role in FHB resistance remains to be determined.

Acknowledgments This is contribution no. 11-032-J from the Kansas Agricultural Experiment Station, Manhattan, Kansas, USA. This project is partly funded by the US Wheat and Barley Scab Initiative and the National Research Initiative Competitive Grants CAP project 2011-68002-30029 from the USDA National Institute of Food and Agriculture. Mention of trade names or commercial products in this article is solely for the purpose of providing specific information and does not imply recommendation or endorsement by the US Department of Agriculture.

\section{References}

Akhunov E, Nicolet C, Dvorak J (2009) Single nucleotide polymorphism genotyping in polyploid wheat with the Illumina goldengate assay. Theor Appl Genet 119: 507-517

Altschul SF, Gish W, Miller W, Myers EW, Lipman DJ (1990) Basic local alignment search tool. J Mol Biol 215: 403-410

Andersen AL (1948) The development of Gibberella zeae headblight of wheat. Phytopathology 38:595-611

Bai G, Zhou C, Qian C, Ge Y (1989) A study on scab resistance in new wheat cultivars and advanced breeding lines. Jiangsu Agr Sci 7:20-22

Bai G, Kolb F, Shaner G, Domier L (1999) Amplified fragment length polymorphism markers linked to a major quantitative trait locus controlling scab resistance in wheat. Phytopathology 89:343-348

Bai G, Shaner G, Ohm H (2000) Inheritance of resistance to Fusarium graminearum in wheat. Theor Appl Genet 100:1-8

Bai G, Guo P, Kolb FL (2003) Genetic relationships among head blight resistant cultivars of wheat assessed on the basis of molecular markers. Crop Sci 43:498-507
Beales J, Laurie D, Devos K (2005) Allelic variation at the linked AP1 and PhyC loci in hexaploid wheat is associated but not perfectly correlated with vernalization response. Theor Appl Genet 110:1099-1107

Bernardo AN, Bradbury PJ, Ma H, Hu S, Bowden RL, Buckler ES, Bai G (2009) Discovery and mapping of single feature polymorphisms in wheat using Affymetrix arrays. BMC Genomics 10:251

Buerstmayr H, Ban T, Anderson JA (2009) QTL mapping and marker-assisted selection for Fusarium head blight resistance in wheat: a review. Plant Breeding 128:1-26

Cuthbert PA, Somers DJ, Thomas J, Cloutier S, Brule-Babel A (2006) Fine mapping Fhb1, a major gene controlling Fusarium head blight resistance in bread wheat (Triticum aestivum L.). Theor Appl Genet 112:1465-1472

Desjardins AE, Hohn TM (1997) Mycotoxins in plant pathogenesis. Mol Plant-Microbe Interact 10:147-152

Garcés-Claver A, Fellman S, Gil-Ortega R, Jahn M, ArnedoAndrés M (2007) Identification, validation and survey of a single nucleotide polymorphism (SNP) associated with pungency in Capsicum spp. Theor Appl Genet 115: 907-916

Grausgruber H, Lemmens M, Bürstmayr H, Ruckenbauer P (2004) Resistance of 'Chinese Spring' substitution sines carrying chromosomes from 'Cheyenne', 'Hope' and 'Lutescens 62' wheats to head blight caused by Fusarium culmorum. Hereditas 130:57-63

Ha B-K, Hussey RS, Boerma HR (2007) Development of SNP assays for marker-assisted selection of two southern rootknot nematode resistance QTL in soybean. Crop Sci 47: S-73-S-82

Hayashi K, Hashimoto N, Daigen M, Ashikawa I (2004) Development of PCR-based SNP markers for rice blast resistance genes at the Piz locus. Theor Appl Genet 108: $1212-1220$

Jayatilake DV, Bai GH, Dong YH (2011) A novel quantitative trait locus for Fusarium head blight resistance in chromosome 7A of wheat. Theor Appl Genet 22:1189-1198

Jin Q, Waters D, Cordeiro GM, Henry RJ, Reinke RF (2003) A single nucleotide polymorphism (SNP) marker linked to the fragrance gene in rice (Oryza sativa L.). Plant Sci 165:359-364

Kaczorowski KA, Kim K-S, Diers BW, Hudson ME (2008) Microarray-based genetic mapping using soybean nearisogenic lines and generation of SNP markers in the Rag1 aphid-resistance interval. Plant Gen 1:89-98

Koebner RMD, Summers RW (2003) 21st century wheat breeding: plot selection or plate detection? Trends Biotechnol 21:59-63

Konishi S, Izawa T, Lin S, Ebana K, Fukuta Y, Sasaki T, Yano M (2006) A SNP caused loss of seed shattering during rice domestication. Science 312:1392-1396

Kosambi DD (1944) The estimation of map distance from recombination values. Ann Eugen 12:172-175

Kumar R, Qiu J, Joshi T, Valliyodan B, Xu D, Nguyen H (2007) Single feature polymorphism discovery in rice. PLoS One 2:e284

Liu S, Abate ZA, Lu H, Musket T, Davis GL, McKendry AL (2007) QTL associated with Fusarium head blight resistance in the soft red winter wheat Ernie. Theor Appl Genet 115:417-427 
Liu S, Anderson JA (2003) Marker assisted evaluation of Fusarium head blight resistant wheat germplasm. Crop Sci 43:760-766

Liu S, Pumphrey MO, Gill BS, Trick HN, Zhang JX, Dolezel J, Chalhoub B, Anderson JA (2008) Toward positional cloning of Fhb1, a major QTL for Fusarium head blight resistance in wheat. Cereal Res Commun 36:195-201

Ma H-X, Bai G-H, Gill BS, Hart LP (2006) Deletion of a chromosome arm altered wheat resistance to Fusarium head blight and deoxynivalenol accumulation in Chinese Spring. Plant Dis 90:1545-1549

Marshall O (2004) PerlPrimer: cross-platform, graphical primer design for standard, bisulphite and real-time PCR. Bioinformatics 20:2471-2472

McMullen M, Jones R, Gallenberg D (1997) Scab of wheat and barley: a re-emerging disease of devastating impact. Plant Dis 81:1340-1348

Mesterhazy A (1977) Reaction of winter wheat varieties to four Fusarium species. Phytopathol Z 90:104-112

Mochida K, Yamazaki Y, Ogihara Y (2004) Discrimination of homoeologous gene expression in hexaploid wheat by SNP analysis of contigs grouped from a large number of expressed sequence tags. Mol Gen Genomics 270: $371-377$

Rafalski A (2002) Applications of single nucleotide polymorphisms in crop genetics. Curr Opin Plant Biol 5:94-100

Roby J, Nelson JC (2008) QGene 4.0, an extensible Java QTLanalysis platform. Bioinformatics 24:2788-2789

Rohlf FJ (1998) NTSYSpc: numerical taxonomy system, ver. 2.02i. Exeter Publishing, Ltd, Setauket, NY

Saghai-Maroof M, Soliman K, Jorgensen R, Allard R (1984) Ribosomal DNA spacer length polymorphism in barley: Mendelian inheritance, chromosomal location, and population dynamics. Proc Natl Acad Sci USA 81:8014-8018

Sattarzadeh A, Achenbach U, Lübeck J, Strahwald J, Tacke E, Hofferbert H-R, Rothsteyn T, Gebhardt C (2006) Single nucleotide polymorphism (SNP) genotyping as basis for developing a PCR-based marker highly diagnostic for potato varieties with high resistance to Globodera pallida pathotype Pa2/3. Mol Breed 18:301-312

Schroeder H, Christensen J (1963) Factors affecting resistance of wheat to scab caused by Gibberella zeae. Phytopathology 53:831-838

Schwieger F, Tebbe CC (1998) A new approach to utilize PCR-single-strand-conformation polymorphism for $16 \mathrm{~S}$ rRNA gene-based microbial community analysis. Appl Environ Microbiol 64:4870-4876

Smith D, Flavell R (1975) Characterization of the wheat genome by renaturation kinetics. Chromosoma 50:223-242

Sneller CH, Garcia G, Gupta A, Nolan R, Lipps P, Herald L, Johnston A (2004) Genetics of FHB resistance in the soft red winter wheat cultivar "Freedom". In: Proceedings of the 2nd international symposium on Fusarium head blight. Orlando, FL, p 166

Snijders CHA (1990) Genetic variation for resistance to Fusarium head blight in bread wheat. Euphytica 50:171-179

Somers D, Kirkpatrick R, Moniwa M, Walsh A (2003) Mining single-nucleotide polymorphisms from hexaploid wheat ESTs. Genome 46:431-437

Sun X, Bai G, Carver B (2009) Molecular markers for wheat leaf rust resistance gene Lr41. Mol Breed 23:311-321

Thompson JD, Higgins DG, Gibson TJ (1994) CLUSTAL W: improving the sensitivity of progressive multiple sequence alignment through sequence weighting, position-specific gap penalties and weight matrix choice. Nucleic Acids Res 22:4673-4680

Waldron BL, Moreno-Sevilla B, Anderson JA, Stack RW, Frohberg RC (1999) RFLP mapping of QTL for Fusarium head blight resistance in wheat. Crop Sci 39:805-811

Wilcoxson RD, Busch RH, Ozmon EA (1992) Fusarium head blight resistance in spring wheat cultivars. Plant Dis 76 : 658-661

Yu GH, Ma HX, Bai GH, Tang KX (2008a) Single-strand conformational polymorphism markers associated with a major QTL for fusarium head blight resistance in wheat. Mol Biol (Mosk) 42:571-580

Yu JB, Bai GH, Zhou WC, Dong YH, Kolb FL (2008b) Quantitative trait loci for Fusarium head blight resistance in a recombinant inbred population of Wangshuibai/ Wheaton. Phytopathology 98:87-94

Zhang X, Zhou M, Ren L, Bai G, Ma H, Scholten OE, Guo P, Lu W (2004) Molecular characterization of Fusarium head blight resistance from wheat variety Wangshuibai. Euphytica 139:59-64

Zhang JX, Jin Y, Rudd JC, Bockelman HE (2008) New Fusarium head blight resistant spring wheat germplasm identified in the USDA national small grains collection. Crop Sci 48:223-235

Zhou W, Kolb FL, Bai G, Domier LL, Yao JB (2002a) Effect of individual Sumai 3 chromosomes on resistance to scab spread within spikes and deoxynivalenol accumulation within kernels in wheat. Hereditas 137:81-89

Zhou W, Kolb FL, Bai G, Shaner Gregory, Domier LL (2002b) Genetic analysis of scab resistance QTL in wheat with microsatellite and AFLP markers. Genome 45:719

Zhou W, Kolb FL, Yu J, Bai G, Boze LK, Domier LL (2004) Molecular characterization of Fusarium head blight resistance in Wangshuibai with simple sequence repeat and amplified fragment length polymorphism markers. Genome 47:1137-1143

Zhu YL, Song QJ, Hyten DL, Van Tassell CP, Matukumalli LK, Grimm DR, Hyatt SM, Fickus EW, Young ND, Cregan PB (2003) Single-nucleotide polymorphisms in soybean. Genetics 163:1123-1134 